\title{
Polarization-Insensitive Metalenses at Visible Wavelengths
}

\section{Citation}

Khorasaninejad, M., A. Y. Zhu, C. Roques-Carmes, W. T. Chen, J. Oh, I. Mishra, R. C. Devlin, and F. Capasso. 2016. "Polarization-Insensitive Metalenses at Visible Wavelengths." Nano Letters 16 (11): 7229-34. https://doi.org/10.1021/acs.nanolett.6b03626.

\section{Permanent link}

http://nrs.harvard.edu/urn-3:HUL.InstRepos:41371324

\section{Terms of Use}

This article was downloaded from Harvard University's DASH repository, WARNING: This file should NOT have been available for downloading from Harvard University's DASH repository.

\section{Share Your Story}

The Harvard community has made this article openly available.

Please share how this access benefits you. Submit a story.

\section{Accessibility}




\title{
Polarization-Insensitive Metalenses at Visible Wavelengths
}

\author{
M. Khorasaninejad, ${ }^{* \dagger}$ A. Y. Zhu, ${ }^{\dagger}$ C. Roques-Carmes, ${ }^{\dagger, \dagger}$ W. T. Chen, ${ }^{\dagger}$ J. Oh, ${ }^{\dagger, \S}$ I. Mishra, ${ }^{\dagger, \S}$ R. C. Devlin, ${ }^{\dagger}$ \\ and F. Capasso ${ }^{*} \dagger$ \\ ${ }^{\dagger}$ Harvard John A. Paulson School of Engineering and Applied Sciences, Harvard University, Cambridge, Massachusetts 02138, United \\ States \\ \#École Polytechnique, Palaiseau 91120, France \\ ${ }^{\S}$ University of Waterloo, Waterloo, ON N2L 3G1, Canada
}

Supporting Information

\begin{abstract}
In this Letter, we demonstrate highly efficient, polarization-insensitive planar lenses (metalenses) at red, green, and blue wavelengths $(\lambda=660,532$, and $405 \mathrm{~nm})$. Metalenses with numerical apertures (NA) of 0.85 and 0.6 and corresponding efficiencies as high as $60 \%$ and $90 \%$ are achieved. These metalenses are less than $600 \mathrm{~nm}$-thick and can focus incident light down to diffraction-limited spots as small as $\sim 0.64 \lambda$ and provide high-resolution imaging. In addition, the focal spots are very symmetric with high Strehl ratios. The single step lithography and compatibility with large-scale fabrication processes make metalenses highly promising for widespread applications in imaging and spectroscopy.
\end{abstract}

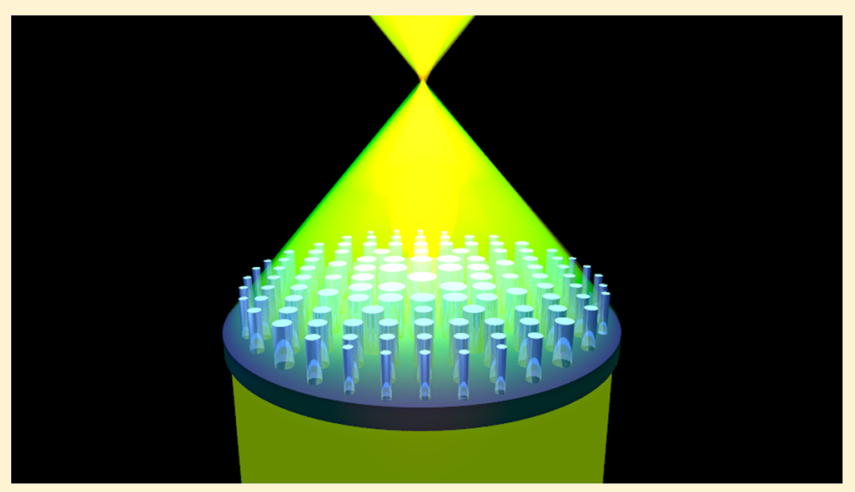

KEYWORDS: Metasurface, polarization-insensitive metalenses, visible wavelength, titanium dioxide

$\mathrm{M}$ etasurfaces $^{1-6}$ have emerged as one of the leading platforms for the development of miniaturized optical components. Although there has been considerable effort in the development of metalenses, ${ }^{7-30}$ their efficient operation range has been mostly limited to the near IR. Achieving highly efficient metalenses in the visible spectrum poses a plethora of challenges mostly arising from the intrinsic optical loss of the constituent materials, which usually consist of either silicon $^{21,31-37}$ or plasmonic noble metals. ${ }^{38-42}$ One can alternatively use dielectrics with a transparency window in the visible spectrum; however, achieving high aspect ratio subwavelength structures with vertical sidewalls is typically very challenging for these materials using current top-down fabrication processes, i.e., lithography followed by dry etching. This results in performance degradation. ${ }^{43}$ It is worth noting that an earlier work on titanium dioxide $\left(\mathrm{TiO}_{2}\right)$ diffractive elements used a dry etching process to fabricate blazed gratings at visible wavelengths $(633 \mathrm{~nm})$. They also demonstrated a $\mathrm{TiO}_{2}$-based lens $(\mathrm{NA}=0.25)$ in the near $\operatorname{IR}(\lambda=860 \mathrm{~nm})^{44}$ but experienced similar difficulties with tapered wall profiles and surface roughness, which are commonly associated with this process. The latter contributes to scattering losses, and the former results in errors in the realized phase. Recently, we have developed a distinct fabrication approach based on the atomic layer deposition (ALD) of amorphous $\mathrm{TiO}_{2}{ }^{45}$ yielding high aspect ratio nanostructures. In particular, the use of $\mathrm{ALD}^{45,46}$ side-steps the aforementioned difficulties with dry etching and allows for high quality amorphous $\mathrm{TiO}_{2}$ with negligible material and scattering loss. Based on this process, we have recently reported geometric phase-based metalenses in the visible spectrum, which require circularly polarized (CP) incident light. $^{47,48}$ In this work, we demonstrate transmissive polarization-insensitive planar lenses (metalenses) operating at red $(660 \mathrm{~nm})$, green $(532 \mathrm{~nm})$, and blue $(405 \mathrm{~nm})$ wavelengths. Metalenses with numerical apertures (NA) of 0.6 and 0.85 are demonstrated with focusing efficiencies up to $90 \%$ and $60 \%$, respectively.

Design of Metalenses. The building blocks of a metalens are $\mathrm{TiO}_{2}$ nanopillars on a glass substrate (Figure 1a). The metalens focuses collimated incident light into a spot in transmission mode. To accomplish this, each nanopillar at position $(x, y)$ must impart the required phase given by

$$
\phi_{t}(x, y)=2 \pi-\frac{2 \pi}{\lambda_{d}}\left(\sqrt{x^{2}+y^{2}+f^{2}}-f\right)
$$

where $\lambda_{d}$ is the design wavelength and $f$ is the focal length. The required phase profile $\varphi_{t}(x, y)$ is realized by adjusting the nanopillar diameter. ${ }^{26,44,49}$ To ensure high efficiency, other parameters such as nanopillar height $H$ and unit cell size $U$ (Figure $1 \mathrm{~b}, \mathrm{c}$ ) are optimized at the design wavelength $\lambda_{d}$. In this design, the phase accumulation is realized by means of the waveguiding effect; the height of the nanopillars should be tall enough to provide $2 \pi$ phase coverage through a range of

Received: August 28, 2016

Revised: October 15, 2016

Published: October 24, 2016 

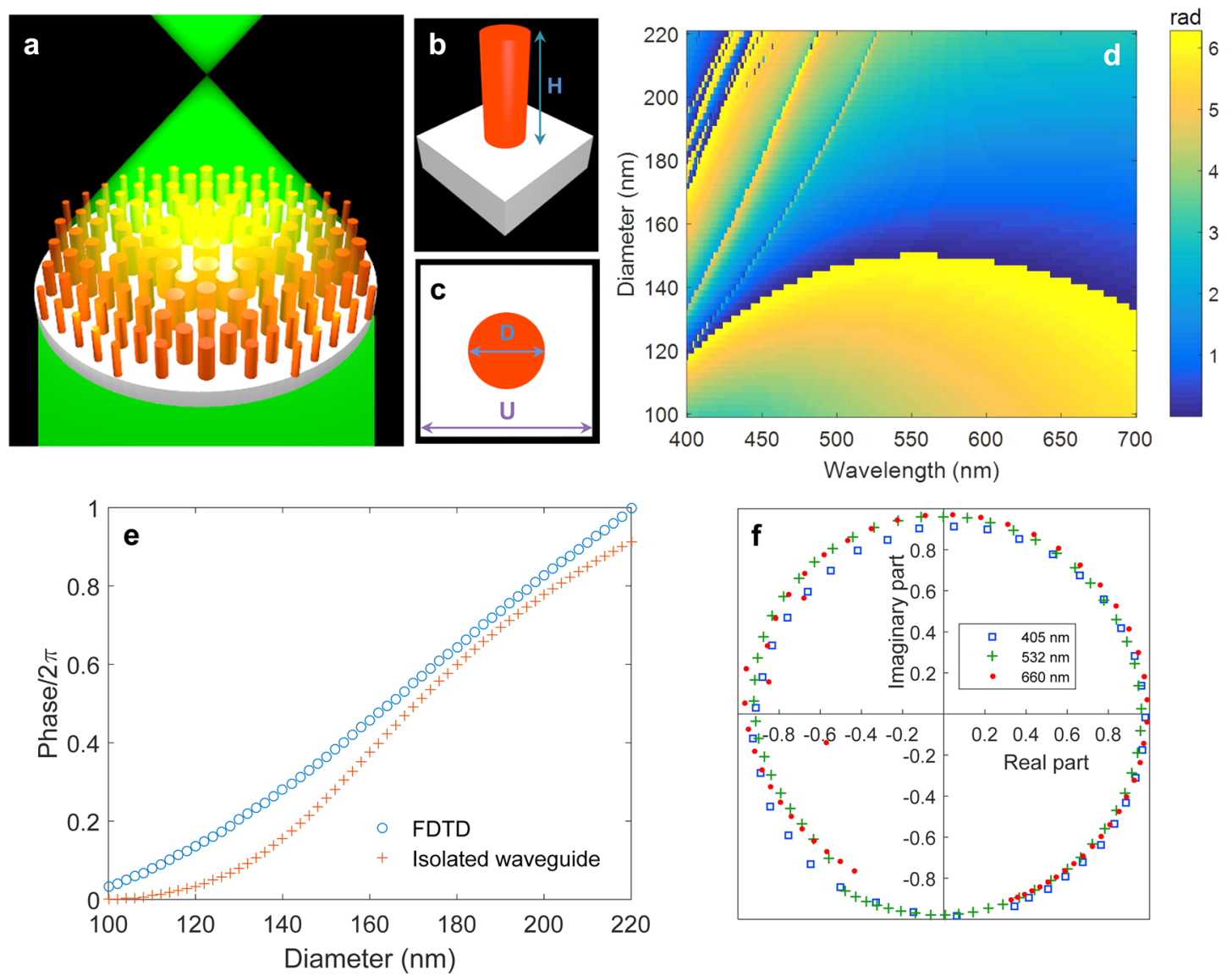

Figure 1. (a) Schematic of a metalens operating in transmission mode. (b,c) Side-view and top-view of the metalens building block: a TiO ${ }_{2}$ nanopillar on a glass substrate. Here we arranged nanopillars in a square lattice. For the design wavelength $\lambda_{d}=405 \mathrm{~nm}\left(\lambda_{d}=532 \mathrm{~nm} ; \lambda_{d}=660 \mathrm{~nm}\right)$, the unit cell dimension is $U=180 \mathrm{~nm}(U=250 \mathrm{~nm} ; U=350 \mathrm{~nm})$, the nanopillar height is $H=400 \mathrm{~nm}(H=600 \mathrm{~nm} ; H=600 \mathrm{~nm})$, and the nanopillar diameters $D$ vary between 80 to $155 \mathrm{~nm}(100 \mathrm{~nm}<D<220 \mathrm{~nm} ; 100 \mathrm{~nm}<D<320 \mathrm{~nm})$. (d) Simulated phase map $\varphi(D)$ for the metalens designed at $\lambda_{d}=532 \mathrm{~nm}$. Each point on this phase map shows the relative phase difference between a nanopillar with diameter $D$ and a reference point where there is no nanopillar (propagation through the air). (e) Comparison of the phase calculated by finite difference time domain simulation of the building block (nanopillar on a glass substrate) as a function of diameter $D$, and the phase due to propagation in an isolated cylindrical waveguide, considering just its fundamental mode $H E_{11}$ at $\lambda_{d}=532 \mathrm{~nm}$. (f) Complex transmission coefficients for three design wavelengths. Each point represents the amplitude and phase of the transmission of a nanopillar with diameter $D$.

diameters. While the smallest attainable diameter is limited primarily by fabrication constraints, the largest one is equal to the unit cell size $U$, which in turn must be small enough to meet the Nyquist sampling criterion $\left(U<\frac{\lambda}{2 N A}\right)$. First, we designed a metalens at the wavelength $\lambda_{d}=532 \mathrm{~nm}$ with nanopillars having height $H=600 \mathrm{~nm}$ and unit cell size $U=$ $250 \mathrm{~nm}$. Figure $1 \mathrm{~d}$ and Figure $S 1$ show the phase map $\varphi(D)$ and transmission map $\mathrm{T}(D)$ as a function of diameter, respectively, across the visible spectrum. Full phase coverage $(2 \pi)$ with high transmission $(>87 \%)$ is achieved.

To gain a better insight into the phase realization mechanism, we calculated the phase imparted solely by the waveguiding effect. This phase is given by

$$
\phi_{W G}=\frac{2 \pi}{\lambda_{d}} n_{e f f} H
$$

where $n_{\text {eff }}$ is the effective index of the fundamental mode $\left(H E_{11}\right)$ and $H$ (nanopillar height) is the propagation length. The $n_{\text {eff }}$ can be readily computed using a single step-index circular waveguide model. ${ }^{50}$ As shown in Figure 1e, the phase calculated by this model follows the one calculated via a finite difference time domain (FDTD) analysis of a nanopillar on a glass substrate. The agreement is better for larger diameters, where the confinement of the fundamental mode increases. While we neglect the confinement along the propagation direction (standing wave due to reflections at both facets of the nanopillars) the average absolute difference between phases calculated using the waveguiding effect and the full-wave analysis is less than $\frac{\pi}{6}$. Note also that in the FDTD calculation periodic boundary conditions are used. They introduce a variation in the mode profile; this effect is larger for smaller diameters, which explains why the phase difference calculated by the two methods is more pronounced in this case. This indicates that the waveguiding effect is the dominant mechanism accounting for the phase realization.

By varying the diameters of nanopillars as a function of their position $\left(x_{i} y_{i}\right)$ the effective index of the propagating mode is changed to achieve the desired phase profile (eq 1). To build the metalens, we discretized its required phase mask $\varphi_{t}\left(x_{i} y_{i}\right)$ assuming square lattice unit cells of dimensions $U \times U$. At each position $\left(x_{i}, y_{i}\right)$ an appropriate diameter, which minimizes I $T_{m} \mathrm{e}^{i \varphi_{t}(x i, y i)}-T(D) \mathrm{e}^{i \varphi(D)} \mid$ is chosen, ${ }^{26}$ where $T_{m}$ is the transmission averaged over all the diameters. Figure if shows the complex transmission coefficients $\left(T(D) \mathrm{e}^{\mathrm{i} \varphi(D)}\right)$ at the three 

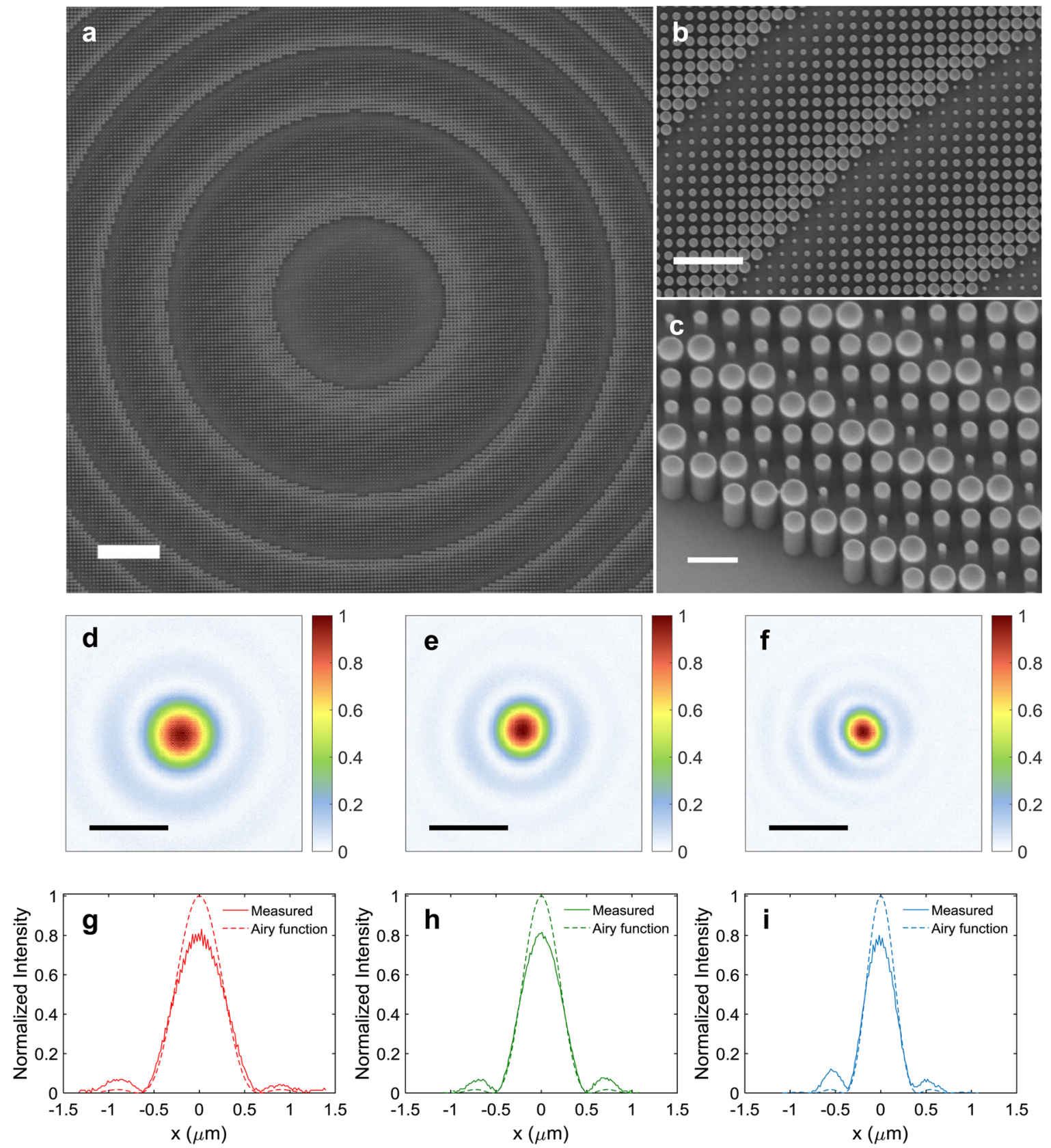

Figure 2. (a) Top-view scanning electron microscope (SEM) image of the center portion of a fabricated metalens. Scale bar: $6 \mu \mathrm{m}$. (b) Top-view SEM image of a portion of the metalens at a higher magnification than that in (a), displaying each individual nanopillar. Scale bar: $2 \mu \mathrm{m}$. (c) Sideview SEM image of the edge of the metalens, showing the vertical profile of the nanopillars. Scale bar: $600 \mathrm{~nm}$. (d-f) Measured focal spots of the metalenses at their design wavelengths of (d) 660, (b) 532, and (c) $405 \mathrm{~nm}$, respectively. Scale bar: $1 \mu \mathrm{m}$. (g-i) Corresponding horizontal cuts of focal spots shown in $\mathrm{d}-\mathrm{f}$ with full width at half-maxima of 633,497 , and $385 \mathrm{~nm}$, respectively. An ideal Airy function is overlaid onto each horizontal cut. All metalenses have diameters of $300 \mu \mathrm{m}$ and focal lengths of $200 \mu \mathrm{m}$, giving numerical apertures NA $=0.6$.

design wavelengths for a range of diameters required to give $2 \pi$ phase coverage. Each point in the complex plane represents the amplitude and phase of the transmission of a nanopillar with diameter $D$, for a given unit cell size and nanopillar height at the corresponding design wavelength. High transmission (with small modulation over the range of used diameters) and close to $2 \pi$ phase coverage is evident for all three design wavelengths.

Fabrication and Characterization. We first fabricated three separate metalenses with identical NA $=0.6$ designed at wavelengths of 405, 532, and $660 \mathrm{~nm}$. Scanning electron microscope images of a fabricated metalens are shown in Figure $2 \mathrm{a}-\mathrm{c}$. Details of fabrication can be found in our previous work. ${ }^{45}$ We characterized these metalenses using a custom-built setup whose schematic is shown in Figure S2a,b. Measured focal spots and their corresponding horizontal cuts are shown in Figure $2 \mathrm{~d}-\mathrm{f}$ and Figure $2 \mathrm{~g}-\mathrm{i}$, respectively. To calculate the Strehl ratio, the measured intensities of the horizontal cuts are normalized to those of ideal Airy functions with the same area under the curve. Airy functions with a maximum intensity of unity and diffraction-limited full width at half-maximum FWHM $=0.514 \frac{\lambda_{d}}{N A}$ are plotted over the horizontal cuts. Strehl ratios of $0.8,0.82$, and 0.83 are achieved at wavelengths of 405 , 532, and $660 \mathrm{~nm}$, respectively. Corresponding Strehl ratios for 

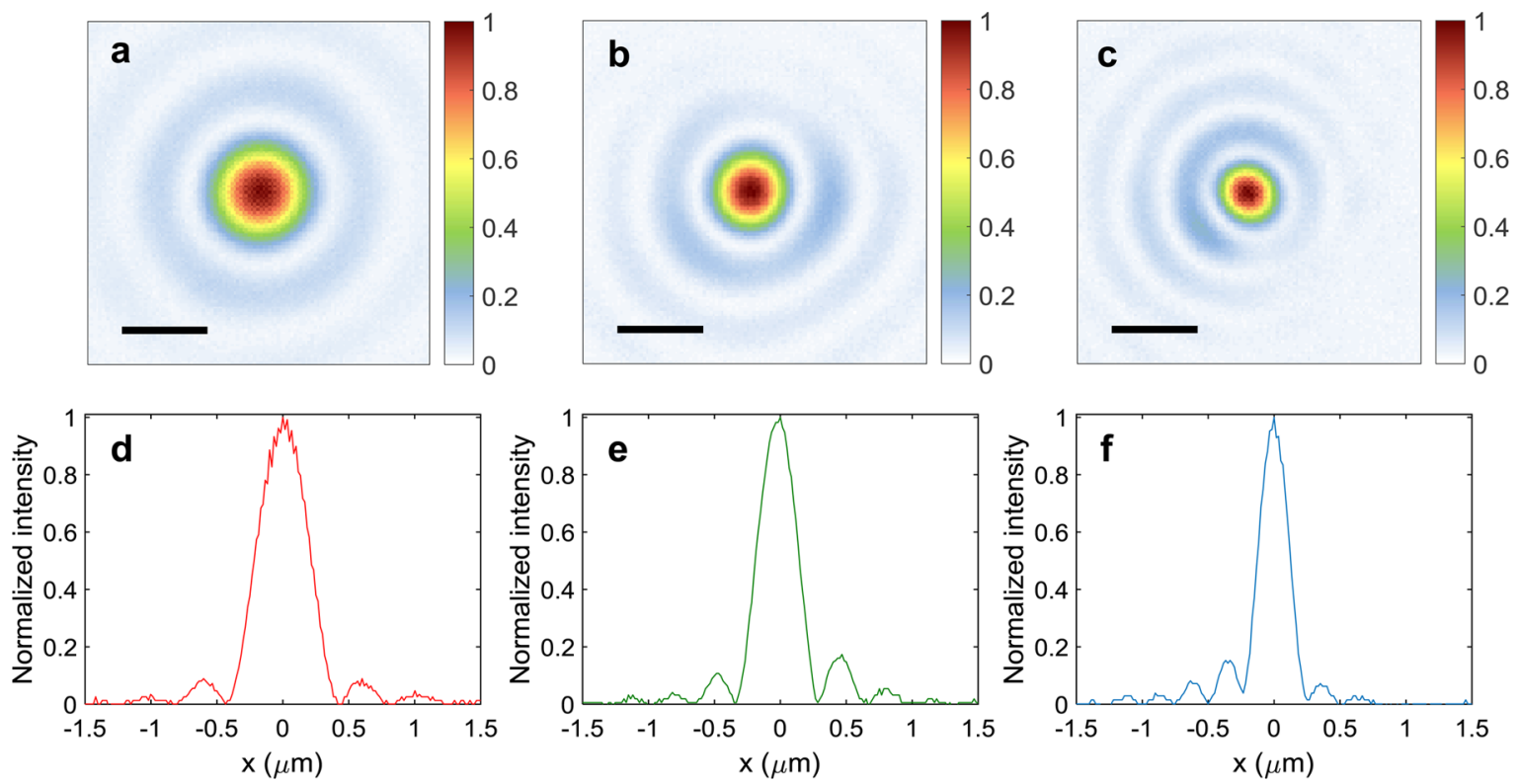

Figure 3. $(\mathrm{a}-\mathrm{c})$ Measured focal spots of NA $=0.85$ metalenses designed at wavelengths of (a) 660 , (b) 532 , and (c) $405 \mathrm{~nm}$. Scale bar: $500 \mathrm{~nm}$. (df) Corresponding horizontal cuts of focal spots in a-c. All metalenses have diameters of $300 \mu \mathrm{m}$ and focal lengths of $90 \mu \mathrm{m}$.
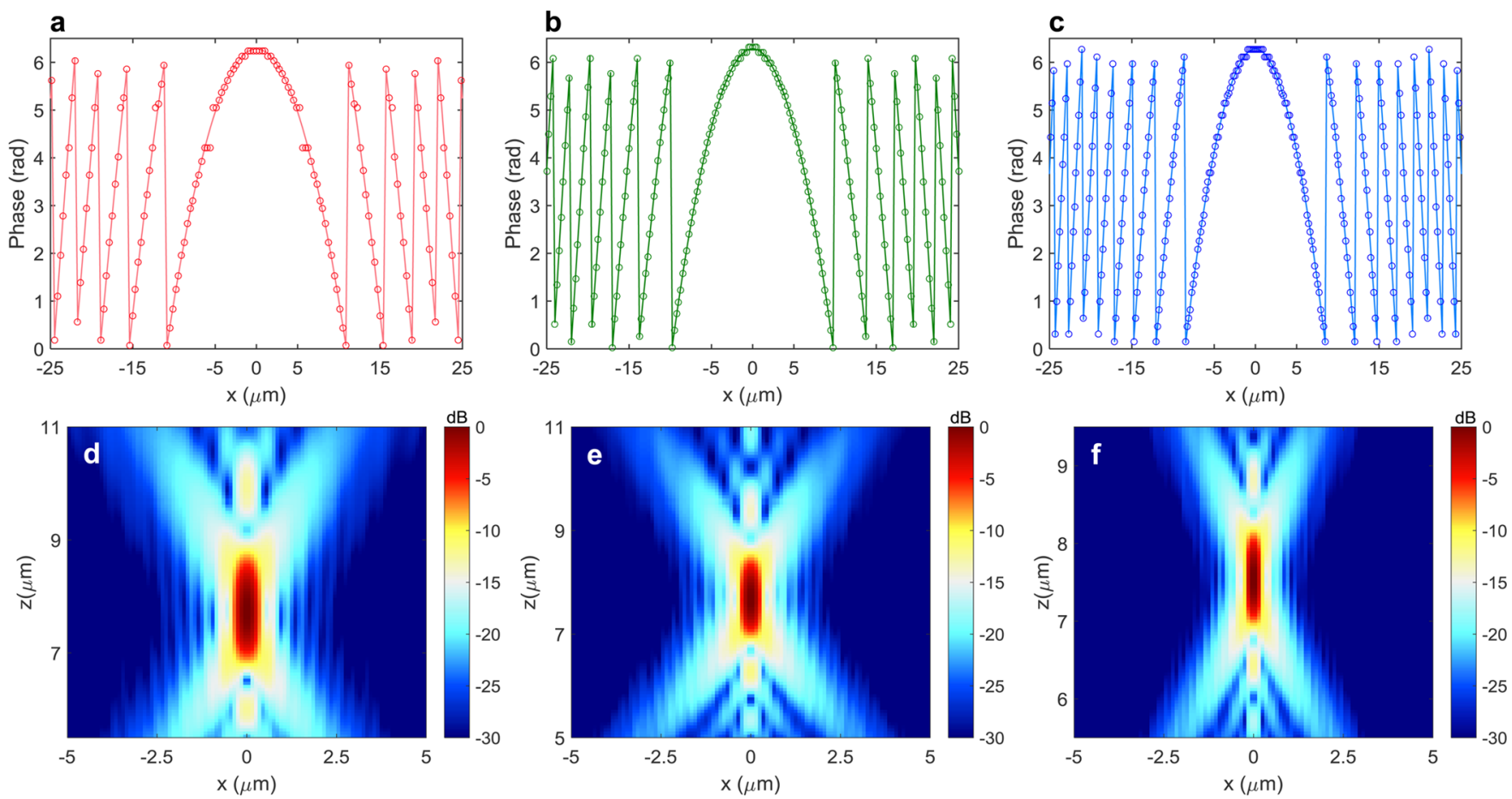

Figure 4. $(a-c)$ Solid lines: required analog phase (eq 1). Circles: digitized phase profiles from FDTD simulations for metalenses designed at wavelengths of (a) 660, (b) 532, and (c) $405 \mathrm{~nm}$. For ease of visualization the phase profiles are shown from $x=-25 \mu \mathrm{m}$ to $x=+25 \mu \mathrm{m}$. Simulated intensity profiles ( $x-z$ plane) in the focal region for metalenses designed at wavelengths of (d) 660, (e) 532, and (f) $405 \mathrm{~nm}$.

vertical cuts (not shown here) are $0.81,0.84$, and 0.81 , which are close to those for horizontal cuts revealing the symmetry of the focal spots. In addition, measured focusing efficiencies as high as $30 \%, 70 \%$, and $90 \%$ are obtained for metalenses designed at wavelengths of 405,532 , and $660 \mathrm{~nm}$, respectively (Figure S3a). The efficiency is calculated as the ratio of the optical power of the measured focused beam to that of the incident beam. The incident beam was measured as the optical power passing through a circular aperture (aluminum on glass) with the same diameter $(300 \mu \mathrm{m})$ as the metalenses.
We subsequently fabricated high $\mathrm{NA}=0.85$ metalenses at the same design wavelengths. Very symmetric focal spots (Figure $3 a-c)$ with diffraction-limited FWHMs are achieved. Figure $3 \mathrm{~d}-\mathrm{f}$ shows the horizontal cuts of focal spots. FWHMs of the horizontal (vertical) cuts of these focal spots are $259 \mathrm{~nm}(256$ $\mathrm{nm}), 327 \mathrm{~nm}(344 \mathrm{~nm})$, and $424 \mathrm{~nm}(428 \mathrm{~nm})$ for metalenses at their respective design wavelengths of 405, 532, and $660 \mathrm{~nm}$. The on-axis modulation transfer function of the metalens $\left(\lambda_{\mathrm{d}}=\right.$ $532 \mathrm{~nm}$ ) also follows that of a diffraction-limited lens (Figure S4). We also calculated the Strehl ratios from the measured 
horizontal (vertical) cuts and obtained values of $0.76(0.78)$, $0.82(0.84)$, and $0.85(0.85)$ corresponding to metalenses designed at wavelengths of 405, 532, and $660 \mathrm{~nm}$. The diffraction-limited focusing and high Strehl ratios confirm the quality of fabrication and capability of this $\mathrm{TiO}_{2}$-based platform to realize high performance planar lenses in the visible spectrum.

The measured efficiencies of the metalenses with $\mathrm{NA}=0.85$ are shown in Figure S3b. Efficiencies as high as $60 \%$ are achieved for both metalenses with design wavelengths of 532 and $660 \mathrm{~nm}$. The efficiency drops to $33 \%$ for the metalens designed at $405 \mathrm{~nm}$. This is due to the stricter fabrication tolerance of this design. In other words, nanopillars designed for shorter wavelengths have $2 \pi$ phase coverage over a smaller range of diameters and smaller unit cells, which make fabrication errors more pronounced resulting in a reduced efficiency. For example, the mean value of the nanopillar diameters used to build the metalens designed at $405 \mathrm{~nm}$ is 120 $\mathrm{nm}$, whereas it is $215 \mathrm{~nm}$ for the one designed at $660 \mathrm{~nm}$.

Figure $4 a-c$ show the required phase profile to realize metalenses $(\mathrm{NA}=0.85)$ at the three design wavelengths $\left(\lambda_{d}=\right.$ 660,532 , and $405 \mathrm{~nm}$ ). We also overlaid the discretized FDTD simulated phases that are theoretically available with suitable nanopillar diameters. Very good fits are obtained for all three designs. We further confirm this by performing FDTD analysis on metalenses with similar $\mathrm{NA}=0.85$ but smaller lens diameters $(24 \mu \mathrm{m})$ than the fabricated ones due to limited computational resources. Figure $4 \mathrm{~d}-\mathrm{f}$ shows the intensity distribution of metalenses in the focal region $(x-z$ plane). We define the efficiency as the ratio of the optical power in the focal spot area (circle of radius $2 \times$ FWM spanning the center of the focal spot) to the incident optical power. Efficiencies as high as $79 \%, 83 \%$, and $84 \%$ are achieved for metalenses designed at wavelengths of 405,532 , and $660 \mathrm{~nm}$, respectively. These simulations set the maximum achievable limit of our design, which can be experimentally obtained by further optimizing the fabrication process, in particular, by overcoming the proximity effect of electron beam lithography. It is notable that tapered nanopillars, which are prevalent in current topdown approaches such as reactive ion etching (RIE) ${ }^{43,44}$ make it more challenging to achieve diffraction-limited focusing and high efficiency (Figure S5).

We also examined the polarization-insensitivity of the metalens designed at $532 \mathrm{~nm}$ by measuring its focal spot and efficiency for different linearly polarized inputs. As shown in Figure S6, very small changes in the shape and size of the focal spots were observed. The standard deviation of the FWHMs of measured focal spots for the different polarizations was $\sim 8 \mathrm{~nm}$. In addition, the change in focusing efficiency for different linearly polarized inputs was negligible.

Imaging. For imaging purposes, we fabricated a new metalens with a diameter of $2 \mathrm{~mm}$ designed at $\lambda_{d}=532 \mathrm{~nm}$. We used the 1951 United States Air Force (USAF) resolution test chart (Thorlabs Inc.) to discern the resolving power of the metalens. Images of the smallest three-bar groups on the chart (bar width of $2.2 \mu \mathrm{m}$ and center-to-center distance of $4.4 \mu \mathrm{m}$ ) are formed by the metalens at different illumination wavelengths and are shown in Figure 5a-d. From this experiment, it is evident that the metalens can resolve micron-sized features across the visible spectrum. The differences in the image size at various wavelengths comes from the fact that the focal length of the metalens is a function of wavelength resulting in a wavelength-dependent magnification. For example, the magni-
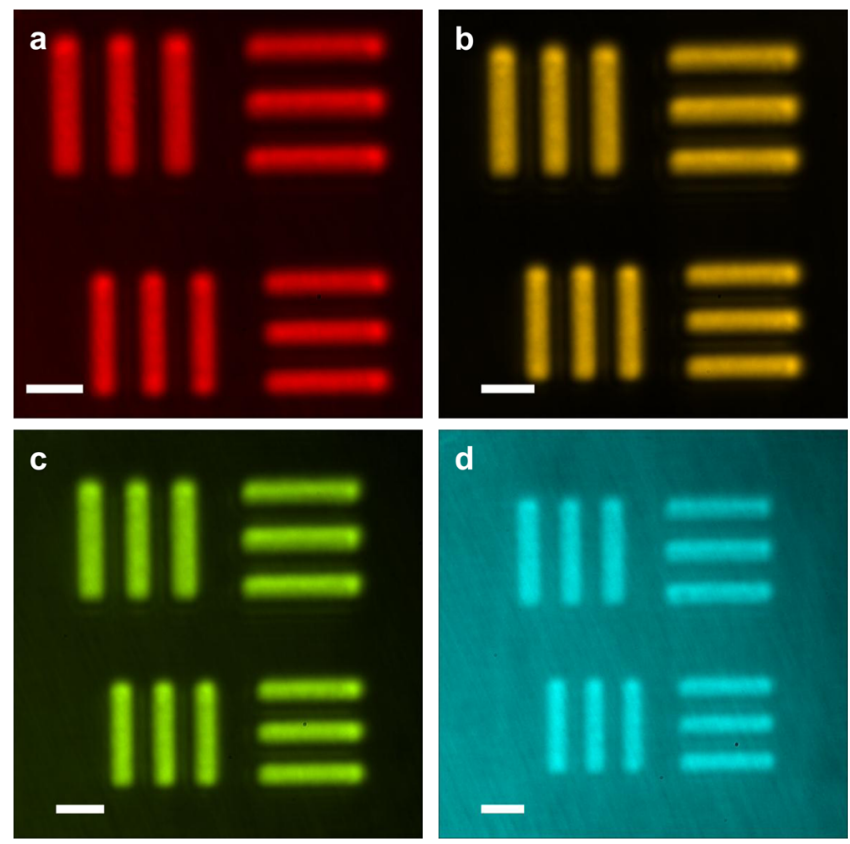

Figure 5. Imaging with the metalens with a diameter of $2 \mathrm{~mm}$ and focal length $f=725 \mu \mathrm{m}$. Images formed by the metalens under an illumination wavelength of (a) 650, (b) 600, (c) 550, and (d) $490 \mathrm{~nm}$. Illumination was provided by a tunable laser (SuperK Varia) with its bandwidth set to $10 \mathrm{~nm}$. Scale bars: $5 \mu \mathrm{m}$.

fication changes from $260 \times$ to $337 \times$ by varying the wavelength of incidence from 490 to $650 \mathrm{~nm}$.

In summary, we have demonstrated metalenses with high efficiency and high NA operating in the visible spectrum. In addition to their capability to focus light into diffraction-limited spots, these metalenses have high resolving power when used for imaging. The ultrathin and compact features of these planar metalenses together with their straightforward fabrication process can play an essential role to achieve miniaturized and lightweight optical systems. We envision this technology will enable a host of cost-effective solutions for versatile applications ranging from imaging, spectroscopy, and laser fabrication to wearable optics.

\section{ASSOCIATED CONTENT}

\section{S Supporting Information}

The Supporting Information is available free of charge on the ACS Publications website at DOI: 10.1021/acs.nanolett.6b03626.

Additional figures (PDF)

\section{AUTHOR INFORMATION}

\section{Corresponding Authors}

*E-mail: khorasani@seas.harvard.edu.

*E-mail: capasso@seas.harvard.edu.

Notes

The authors declare no competing financial interest.

\section{ACKNOWLEDGMENTS}

This work was supported in part by the Air Force Office of Scientific Research (MURI, grant\# FA9550-14-1-0389 and FA9550-16-1-0156) and Thorlabs Inc. W.T.C. acknowledges postdoctoral fellowship support from the Ministry of Science and Technology, Taiwan (104-2917-I-564-058). A.Y.Z. thanks 
Harvard SEAS and A*STAR Singapore under the National Science Scholarship scheme. R.C.D. is supported by a Charles Stark Draper Fellowship. This work was performed in part at the Center for Nanoscale Systems (CNS), a member of the National Nanotechnology Coordinated Infrastructure (NNCI), which is supported by the National Science Foundation under NSF award no. 1541959. CNS is a part of Harvard University. We thank E. Hu for the supercontinuum laser (NKT "SuperK"). We also thank S. Byrnes, N. Rubin, and V. Sanjeev for their help and useful discussion.

\section{REFERENCES}

(1) Yu, N.; Capasso, F. Nat. Mater. 2014, 13 (2), 139-150.

(2) Kildishev, A. V.; Boltasseva, A.; Shalaev, V. M. Science 2013, 339 (6125), 1232009.

(3) Falcone, F.; Lopetegi, T.; Laso, M.; Baena, J.; Bonache, J.; Beruete, M.; Marqués, R.; Martin, F.; Sorolla, M. Phys. Rev. Lett. 2004, 93 (19), 197401.

(4) Zheludev, N. I.; Kivshar, Y. S. Nat. Mater. 2012, 11 (11), 917924.

(5) Holloway, C. L.; Kuester, E. F.; Gordon, J. A.; O’Hara, J.; Booth, J.; Smith, D. R. IEEE Antennas Propag. Mag. 2012, 54 (2), 10-35.

(6) Glybovski, S. B.; Tretyakov, S. A.; Belov, P. A.; Kivshar, Y. S.; Simovski, C. R. Phys. Rep. 2016, 634, 1-72.

(7) Levy, U.; Abashin, M.; Ikeda, K.; Krishnamoorthy, A.; Cunningham, J.; Fainman, Y. Phys. Rev. Lett. 2007, 98 (24), 243901.

(8) Grbic, A.; Jiang, L.; Merlin, R. Science 2008, 320 (5875), 511513.

(9) Verslegers, L.; Catrysse, P. B.; Yu, Z.; White, J. S.; Barnard, E. S.; Brongersma, M. L.; Fan, S. Nano Lett. 2009, 9 (1), 235-238.

(10) Huang, F. M.; Kao, T. S.; Fedotov, V. A.; Chen, Y.; Zheludev, N. I. Nano Lett. 2008, 8 (8), 2469-2472.

(11) Lerman, G. M.; Yanai, A.; Levy, U. Nano Lett. 2009, 9 (5), 2139-2143.

(12) Fattal, D.; Li, J.; Peng, Z.; Fiorentino, M.; Beausoleil, R. G. Nat. Photonics 2010, 4 (7), 466-470.

(13) Chang-Hasnain, C. J. Semicond. Sci. Technol. 2011, 26 (1), 014043.

(14) Schonbrun, E.; Seo, K.; Crozier, K. B. Nano Lett. 2011, 11 (10), 4299-4303.

(15) Zentgraf, T.; Liu, Y.; Mikkelsen, M. H.; Valentine, J.; Zhang, X. Nat. Nanotechnol. 2011, 6 (3), 151-155.

(16) Aieta, F.; Genevet, P.; Kats, M. A.; Yu, N.; Blanchard, R.; Gaburro, Z.; Capasso, F. Nano Lett. 2012, 12 (9), 4932-4936.

(17) Rogers, E. T.; Lindberg, J.; Roy, T.; Savo, S.; Chad, J. E.; Dennis, M. R.; Zheludev, N. I. Nat. Mater. 2012, 11 (5), 432-435.

(18) Chen, X.; Huang, L.; Mühlenbernd, H.; Li, G.; Bai, B.; Tan, Q.; Jin, G.; Qiu, C.-W.; Zhang, S.; Zentgraf, T. Nat. Commun. 2012, 3, 1198.

(19) Monticone, F.; Estakhri, N. M.; Alù, A. Phys. Rev. Lett. 2013, 110 (20), 203903.

(20) West, P. R.; Stewart, J. L.; Kildishev, A. V.; Shalaev, V. M.; Shkunov, V. V.; Strohkendl, F.; Zakharenkov, Y. A.; Dodds, R. K.; Byren, R. Opt. Express 2014, 22 (21), 26212-26221.

(21) Lin, D.; Fan, P.; Hasman, E.; Brongersma, M. L. Science 2014, 345 (6194), 298-302.

(22) Cheng, J.; Ansari-Oghol-Beig, D.; Mosallaei, H. Opt. Lett. 2014, 39 (21), 6285-6288.

(23) Aieta, F.; Kats, M. A.; Genevet, P.; Capasso, F. Science 2015, 347 (6228), 1342-1345.

(24) Eisenbach, O.; Avayu, O.; Ditcovski, R.; Ellenbogen, T. Opt. Express 2015, 23 (4), 3928-3936.

(25) Pors, A.; Nielsen, M. G.; Bozhevolnyi, S. I. Optica 2015, 2 (8), $716-723$.

(26) Arbabi, A.; Horie, Y.; Ball, A. J.; Bagheri, M.; Faraon, A. Nat. Commun. 2015, 6, 7069.
(27) Khorasaninejad, M.; Aieta, F.; Kanhaiya, P.; Kats, M. A.; Genevet, P.; Rousso, D.; Capasso, F. Nano Lett. 2015, 15 (8), 53585362 .

(28) Khorasaninejad, M.; Ambrosio, A.; Kanhaiya, P.; Capasso, F. Sci. $A d v .2016,2$ (5), e1501258.

(29) Khorasaninejad, M.; Chen, W. T.; Oh, J.; Capasso, F. Nano Lett. 2016, 16, 3732-3737.

(30) Wang, Q.; Rogers, E. T.; Gholipour, B.; Wang, C.-M.; Yuan, G.; Teng, J.; Zheludev, N. I. Nat. Photonics 2015, 10 (1), 60-65.

(31) Bomzon, Z. e.; Biener, G.; Kleiner, V.; Hasman, E. Opt. Lett. 2002, 27 (5), 285-287.

(32) Spinelli, P.; Verschuuren, M.; Polman, A. Nat. Commun. 2012, 3, 692.

(33) Khorasaninejad, M.; Crozier, K. B. Nat. Commun. 2014, 5, 5386.

(34) Khorasaninejad, M.; Capasso, F. Nano Lett. 2015, 15 (10), 6709-6715.

(35) Arbabi, A.; Horie, Y.; Bagheri, M.; Faraon, A. Nat. Nanotechnol. 2015, 10, 937.

(36) Yu, Y. F.; Zhu, A. Y.; Paniagua-Domínguez, R.; Fu, Y. H.; Luk'yanchuk, B.; Kuznetsov, A. I. Laser Photonics Rev. 2015, 9 (4), 412-418.

(37) Decker, M.; Staude, I.; Falkner, M.; Dominguez, J.; Neshev, D. N.; Brener, I.; Pertsch, T.; Kivshar, Y. S. Adv. Opt. Mater. 2015, 3 (6), 813-820.

(38) Sun, S.; Yang, K.-Y.; Wang, C.-M.; Juan, T.-K.; Chen, W. T.; Liao, C. Y.; He, Q.; Xiao, S.; Kung, W.-T.; Guo, G.-Y. Nano Lett. 2012, 12 (12), 6223-6229.

(39) Yin, X.; Ye, Z.; Rho, J.; Wang, Y.; Zhang, X. Science 2013, 339 (6126), 1405-1407.

(40) Zheng, G.; Mühlenbernd, H.; Kenney, M.; Li, G.; Zentgraf, T.; Zhang, S. Nat. Nanotechnol. 2015, 10 (4), 308-312.

(41) Goh, X. M.; Zheng, Y.; Tan, S. J.; Zhang, L.; Kumar, K.; Qiu, C.W.; Yang, J. K. W. Nat. Commun. 2014, 5, 5361.

(42) Mehmood, M.; Mei, S.; Hussain, S.; Huang, K.; Siew, S.; Zhang, L.; Zhang, T.; Ling, X.; Liu, H.; Teng, J. Adv. Mater. 2016, 28, 2533.

(43) Zhan, A.; Colburn, S.; Trivedi, R.; Fryett, T. K.; Dodson, C. M.; Majumdar, A. ACS Photonics 2016, 3 (2), 209-214.

(44) Lalanne, P.; Astilean, S.; Chavel, P.; Cambril, E.; Launois, H. J. Opt. Soc. Am. A 1999, 16 (5), 1143-1156.

(45) Devlin, R. C.; Khorasaninejad, M.; Chen, W. T.; Oh, J.; Capasso, F. Proc. Natl. Acad. Sci. U. S. A. 2016, 113, 10473.

(46) High, A. A.; Devlin, R. C.; Dibos, A.; Polking, M.; Wild, D. S.; Perczel, J.; de Leon, N. P.; Lukin, M. D.; Park, H. Nature 2015, 522 (7555), 192-196.

(47) Khorasaninejad, M.; Chen, W. T.; Devlin, R. C.; Oh, J.; Zhu, A. Y.; Capasso, F. Science 2016, 352 (6290), 1190-1194.

(48) Khorasaninejad, M.; Chen, W. T.; Zhu, A. Y.; Oh, J.; Devlin, R. C.; Rousso, D.; Capasso, F. Nano Lett. 2016, 16, 4595-4600.

(49) Vo, S.; Fattal, D.; Sorin, W. V.; Peng, Z.; Tran, T.; Fiorentino, M.; Beausoleil, R. G. IEEE Photonics Technol. Lett. 2014, 26 (13), $1375-1378$

(50) Yariv, A.; Yeh, P. Photonics: optical electronics in modern communications (the oxford series in electrical and computer engineering); Oxford University Press, Inc.: 2006. 